AJMS

28,1

44

Received 20 August 2020

Revised 17 December 2020 21 January 2021

Accepted 23 January 2021

\section{Finite extinction for a doubly nonlinear parabolic equation of fast diffusion type}

\author{
Md Abu Hanif Sarkar \\ Department of Mathematics, Kumamoto University, Kumamoto, Japan and \\ Department of Mathematics, Jagannath University, Dhaka, Bangladesh
}

\begin{abstract}
Purpose - The purpose of this paper is to find a doubly nonlinear parabolic equation of fast diffusion in a bounded domain.

Design/methodology/approach - For positive and bounded initial data, the authors study the initial zero-boundary value problem.

Findings - The findings of this study showed the complete extinction of a continuous weak solution at a finite time.

Originality/value - The extinction time is studied earlier but for the Laplacian case. The authors presented the finite extinction time for the case of $p$-Laplacian.
\end{abstract}

Keywords Doubly nonlinear equation, Degenerate and singular equation, Sobolev critical case,

Finite time extinction

\section{Introduction}

Let $\Omega \subset \mathbb{R}^{n}(n \geq 3)$ be a bounded domain with smooth boundary $\partial \Omega$. For any positive $T \leq \infty$, let $\Omega_{T}:=\Omega \times(0, T)$ be the space-time cylinder, and let $\partial_{p} \Omega_{T}$ be the parabolic boundary defined by $(\partial \Omega \times[0, T)) \cup(\Omega \times\{t=0\})$. Throughout this paper, we fix $p \in[2, n)$ and $q+1=p^{*}$, where $p^{*}:=\frac{n p}{n-p}$ is the Sobolev critical exponent. We consider the following doubly nonlinear parabolic equation

$$
\begin{cases}\partial_{t}\left(u^{q}\right)-\Delta_{p} u=0 & \text { in } \Omega_{\infty} \\ u=0 & \text { on } \partial \Omega \times(0, \infty) \\ u(\cdot, 0)=u_{0}(\cdot) & \text { in } \Omega\end{cases}
$$

Here the unknown function $u=u(x, t)$ is a nonnegative real-valued function defined for $(x, t) \in \Omega_{\infty}:=\Omega \times(0, \infty)$, and the initial data $u_{0}$ is assumed to be in the Sobolev space $W_{0}^{1, p}(\Omega)$, positive and bounded in $\Omega$ and, $\Delta_{p} u:=\operatorname{div}\left(|\nabla u|^{p-2} \nabla u\right)$ is the $p$-Laplacian.

First of all, we will recall some fundamental results for the Eqn (1.1).

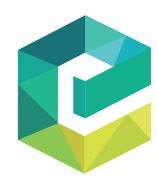

2010 Mathematics Subject Classification — Primary 35B51, 35B65, Secondary 35D30, 35K61.

(C) Md Abu Hanif Sarkar. Published in Arab Journal of Mathematical Sciences. Published by Emerald Publishing Limited. This article is published under the Creative Commons Attribution (CC BY 4.0) licence. Anyone may reproduce, distribute, translate and create derivative works of this article (for both commercial and non-commercial purposes), subject to full attribution to the original publication and authors. The full terms of this licence may be seen at http://creativecommons.org/licences/by/4.0/ legalcode

The author would like to thank the supervisor Prof. Masashi Misawa for his continuous guidance to prepare this paper. Also thanks to Dr Kenta Nakamura for several stimulating discussions. This work is supported by the Grant-in-Aid for Scientific Research (C) No.18K03375 at Japan Society for the Promotion of Science. 
In the case $p=2$, the Eqn (1.1) becomes the so-called porous medium equation or the plasma equation with the Sobolev critical exponent $q+1=\frac{2 n}{n-2}$. The global existence and continuity of a weak solution of (1.1) in the case $p=2$ is proved in [1-3]. In particular, the extinction at a finite time of a continuous weak solution is shown in $[4,5]$. The positivity of the unique weak solution is demonstrated in [6] and the decay estimation has presented in $[6,7]$. The asymptotic behavior at the extinction time is also studied (see $[8,9]$ ). The regularity results for the porous medium equations and the $p$-Laplace equations are also established and are the fundamental theory for the degenerate and singular parabolic equations (see [10, 11]). Here we will consider a doubly nonlinear equation (1.1) with the $p$-Laplacian, the porous medium operator and the Sobolev critical exponent and study the positivity, boundedness and finite time extinction of a weak solution of Eqn (1.1). The Hölder regularity of a weak solution to Eqn (1.1) in homogeneous case $q+1=p$ accomplished in [12]. On the other hand, in the nonhomogeneous case that $q+1$ is not equal to $p$, the regularity of a weak solution is remained to be settled.

A doubly nonlinear parabolic equation, like the one considered above, has been studied before, and the global existence of a weak solution is proved in $[13,14]$ and, for a positive, bounded initial data in the Sobolev space $W_{0}^{1, p}(\Omega)$, the boundedness, the expansion of positivity and regularity of a weak solution to Eqn (1.1) are accomplished in [15] in the Sobolev critical case that $q+1=\frac{n p}{n-p}$. The finite time extinction is also shown in [13]. These results are the starting point of our study in the paper and thus, we will recall these results later.

Now we will explain in details what we mean by complete extinction for solutions of problem in Eqn (1.1). Our aim here is to show that there exists a positive time $T$ such that $u$ is positive in $\Omega \times[0, T)$ and $u=0$ in $\Omega \times[T, \infty)$. This $T$ is called the complete extinction time for Eqn (1.1). From the preceding results, the expansion of positivity and a finite time extinction, obtained in $[13,15]$, we find that a nonnegative weak solution of Eqn (1.1) is positive in $\Omega \times\left[0, T_{0}\right)$ for some $T_{0}>0$ and $u$ vanishes in $\Omega \times\left[T^{*}, \infty\right)$ for some $T^{*}>T_{0}$. Here we notice the possibility that a gap $T_{0}<T^{*}$ may appear and thus, the solution may have positive portion together with zero one in $\Omega \times\left[T_{0}, T^{*}\right)$. The proof of the finite time complete extinction is nothing but to show the equality $T_{0}=T^{*}$, that is the main issue in this paper. Our main assertion is the following:

Theorem 1.1. (Finite time complete extinction). Let $n=3, p \in[2,3)$ and $q+1=\frac{3 p}{3-p}$. Suppose that $\Omega$ is a convex bounded domain. Let $u_{0}$ be positive, bounded and in $W_{0}^{1, p}(\Omega)$. Let $u$ be a nonnegative, continuous weak solution of (1.1). Then there exists a positive $T<\infty$ such that $T$ is the complete extinction time for (1.1), that is, $u$ is positive in $\Omega \times[0, T)$ and $u$ vanishes in $\Omega \times[T, \infty)$.

Under the interior positivity and finite time extinction, explained above, our main task is to devise an appropriate comparison function, rely on the comparison theorem and verify that $T_{0}=T^{*}$ above. We follow the construction of comparison function in [6], where the Laplace operator being $p=2$ is studied in any dimensional space domain. Here we shall treat the doubly nonlinear operator, the $p$-Laplacian coupled with the porous medium operator with the Sobolev critical exponent $q+1=\frac{3 p}{3-p}$ in three dimensional space domain. We shall compute the $p$-Laplacain under polar coordinates in the three space dimension, since the higher dimension case seems to be technically difficult. So far, there is no generalized method to evaluate the $p$-Laplacian in higher dimensional case since this operator is nonlinear and we cannot apply the cylindrical coordinates and the mathematical induction to generalize the case for higher dimension. The convexity of domain is used for the comparison argument to be worked well for our demand (see the proof of Theorem 4.1). Here we also need to assume the continuity of a weak solution to Eqn (1.1), because the regularity for (1.1) is now unknown to
Equation of fast diffusion 
AJMS

28,1

46 be valid in the nonhomogeneous case that $q+1$ is not equal to $p$, stated as before. In the forthcoming work, we shall study how to remove the assumption of regularity.

The structure of this paper is as follows. In Section 2, we prepare some notations, algebraic inequalities and the definition of weak solution for future use. In Section 3, we gather the fundamental properties of a weak solution of Eqn (1.1) such as the global existence of a weak solution, nonnegativity and boundedness, the so-called expansion of positivity and a finite time extinction of a weak solution. The final Section 4 is devoted to the main theorem and its proof. The proof relies on an appropriate choice of comparison function. Here the computation of the $p$-Laplace operator is done under the polar-coordinates in three dimensional space domain.

Remark 1.2. We prove our main theorem for the case of critical Sobolev exponent as we have used the Talenti's function which is a unique solution of the stationary equation on all of space $\mathbb{R}^{n}$ corresponding to Eqn $(1.1)$ (see $[16,17]$ ) to make bound the extinction time.This special function is legitimate for the case of critical Sobolev exponent. Using the usual energy estimates finite extinction time for the subcritical and critical case can be achieved (see [18]). For the supercritical case finite extinction time cannot work well.

\section{Preliminaries}

We exhibit in this section some notation, analytic tools, definition and statement of some basic theorems including the comparison theorem used later.

\subsection{Notation}

Following the standard text books $[19,20]$, we set the following notation. Let $\Omega$ be a bounded domain in $\mathbb{R}^{n}(n \geq 3)$ with smooth boundary $\partial \Omega$ and for a positive $T \leq \infty$ let $\Omega_{T}:=\Omega \times(0, T)$ be the cylindrical domain. Let us define the parabolic boundary of $\Omega_{T}$ by

$$
\partial_{p} \Omega_{T}:=\partial \Omega \times[0, T) \cup \Omega \times\{t=0\} .
$$

Now, we will present some function spaces, defined on space-time region. For $1 \leq p, q \leq \infty, L^{q}\left(t_{1}, t_{2} ; L^{p}(\Omega)\right)$ is a function space of measurable real-valued functions on a space-time region $\Omega \times\left(t_{1}, t_{2}\right)$ with a finite norm

$$
\|v\|_{L^{q}\left(t_{1}, t_{2} ; L^{p}(\Omega)\right)}:= \begin{cases}\left(\int_{t_{1}}^{t_{2}}\|v(t)\|_{L^{p}(\Omega)}^{q} d t\right)^{\frac{1}{q}} & (1 \leq q<\infty) \\ \underset{t_{1} \leq t \leq t_{2}}{\operatorname{ess~sup}\|v(t)\|_{L^{p}(\Omega)}} & (q=\infty),\end{cases}
$$

where

$$
\|v(t)\|_{L^{p}(\Omega)}:= \begin{cases}\left(\int_{\Omega}|v(x, t)|^{p} d x\right)^{\frac{1}{p}} & (1 \leq p<\infty) \\ \underset{x \in \Omega}{\operatorname{ess} \sup |v(x, t)|} & (p=\infty) .\end{cases}
$$

when $p=q$, we write $L^{p}\left(\Omega \times\left(t_{1}, t_{2}\right)\right)=L^{p}\left(t_{1}, t_{2} ; L^{p}(\Omega)\right)$ for brevity. For $1 \leq p<\infty$ the Sobolev space $W^{1, p}(\Omega)$ consists of measurable real-valued functions that are weakly differentiable and their weak derivatives are $p$-th integrable on $\Omega$, with the norm 


$$
\|v\|_{W^{1, p}(\Omega)}:=\left(\int_{\Omega}|v|^{p}+|\nabla v|^{p} d x\right)^{\frac{1}{p}},
$$

where $\nabla v=\left(v_{x_{1}}, \ldots, v_{x_{n}}\right)$ denotes the gradient of $v$ in a distribution sense, and let $W_{0}^{1, p}(\Omega)$

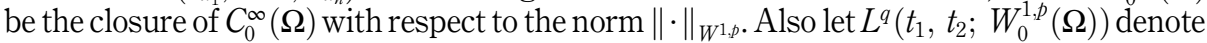
a function space of measurable real-valued functions on space-time region with a finite norm

$$
\|v\|_{L^{q}\left(t_{1}, t_{2} ; W_{0}^{1, \phi}(\Omega)\right)}:=\left(\int_{t_{1}}^{t_{2}}\|v(t)\|_{W^{1, \phi}(\Omega)}^{q} d t\right)^{\frac{1}{q}} .
$$

Let $B=B_{\rho}\left(x_{0}\right):=\left\{x \in \mathbb{R}^{n}:\left|x-x_{0}\right|<\rho\right\}$ denote an open ball with radius $\rho>0$ centered at some $x_{0} \in \mathbb{R}^{n}$.

The algebraic inequality is often used later on.

Lemma 2.1. (Algebraic inequalities, [10]). For every $p \in(1, \infty)$ there exist positive constants $C_{1}(p, n)$ and $C_{2}(p, n)$ such that for any $\xi, \eta \in \mathbb{R}^{n}$

$$
\left.|| \xi\right|^{p-2} \xi-|\eta|^{p-2} \eta\left|\leq C_{1}(|\xi|+|\eta|)^{p-2}\right| \xi-\eta \mid
$$

and

$$
\left(|\xi|^{p-2} \xi-|\eta|^{p-2} \eta\right) \cdot(\xi-\eta) \geq C_{2}|\xi-\eta|^{p}
$$

where dot . denotes the inner product in $\mathbb{R}^{n}$.

\subsection{Weak solution}

Here we are going to define a weak solution which is the most basic prerequisite element to conduct the ongoing research of our Eqn (1.1).

Definition 2.2. Let $0<T \leq \infty$. A measurable function $u$ defined on $\Omega_{T}$ is called a weak supersolution (subsolution) to (1.1) if the following (D1)-(D3) are satisfied:

(D1) $u \in L^{\infty}\left(0, T ; W_{0}^{1, p}(\Omega)\right), \partial_{t}\left(u^{q}\right) \in L^{2}\left(\Omega_{T}\right)$;

(D2) For every nonnegative $\varphi \in C_{0}^{\infty}\left(\Omega_{T}\right)$,

$$
-\int_{\Omega_{T}} u^{q} \varphi_{t} d x d t+\int_{\Omega_{T}}|\nabla u|^{p-2} \nabla u \cdot \nabla \varphi d x d t \geq(\leq) 0 .
$$

(D3) $\left\|u(t)-u_{0}\right\|_{W^{1, \phi}} \rightarrow 0$ as $t \rightarrow 0$.

A measurable function $u$ defined on $\Omega_{T}$ is called a weak solution to Eqn (1.1) if it is simultaneously a weak sub and supersolution, that is,

$$
-\int_{\Omega_{T}} u^{q} \varphi_{t} d x d t+\int_{\Omega_{T}}|\nabla u|^{p-2} \nabla u \cdot \nabla \varphi d x d t=0
$$

for every $\varphi \in C_{0}^{\infty}\left(\Omega_{T}\right)$. 
AJMS

28,1

48

\section{Fundamental facts and results}

In this section, let $n \geq 3, p \in[2, n)$ and $q+1=\frac{n p}{n-p}$, the Sobolev exponent.

\subsection{Existence of a weak solution}

We first state the global existence of a weak solution of Eqn (1.1). For the proof see [14, Theorem 1.1].

Theorem 3.1. (Existence of a weak solution).

For any $u_{0} \in W_{0}^{1, p}(\Omega) \cap L^{\infty}(\Omega)$, there exists a global weak solution of Eqn (1.1).

\subsection{Nonnegativity and boundedness}

We notice that a weak solution to Eqn (1.1) is nonnegative and bounded provided that the initial value $u_{0}$ does so. See [15, Propositions 3.4 and 3.5] for the proof.

Proposition 3.2. (Nonnegativity and boundedness). Suppose that $u_{0} \in W_{0}^{1, \phi}(\Omega)$, nonnegative and bounded in $\Omega$. Then

$$
0 \leq u \leq\left|u_{0}\right|_{L^{\infty}(\Omega)} \text { in } \Omega_{T} .
$$

\subsection{Comparison theorem}

We here state the comparison theorem being available for Eqn (1.1), that is used in the proof of our main theorem later. The proof is given in Appendix. We say that $u \geq v$ on $\partial_{p} \Omega_{T}$ in the trace sense, if

$$
\begin{aligned}
& (-u(t)+v(t))_{+} \in W_{0}^{1, p}(\Omega), \text { for every } t \in(0, T), \\
& \text { and }\left(-u^{q}(t)+v^{q}(t)\right)_{+} \rightarrow 0 \text { in } L^{1}(\Omega) \text { as } t \searrow 0 .
\end{aligned}
$$

Theorem 3.3. (Comparison theorem, [15]). Let $u$ and $v$ be a weak supersolution and subsolution to (1.1) in $\Omega_{T}:=\Omega \times(0, T)$, respectively. If $u \geq v$ on $\partial_{p} \Omega$ in the sense (3.1), then it holds true that

$$
u \geq v \text { in } \Omega_{T} .
$$

\subsection{Expansion of positivity}

In this section, we state some positivity results of a nonnegative weak solution to the doubly nonlinear Eqn (1.1), that we will use later to prove our main theorem. We recall that the socalled expansion of positivity holds true for a nonnegative weak solution of Eqn (1.1). This positivity result is proved in [15]. Here we recall them without the proof. The regularity of the solution can be realized upto the positivity region and after that region we do not know about the regularity of the solution of Eqn (1.1).

The following is the expansion of positivity in a compact subdomain. For the proof see [15, Theorem 4.7].

Theorem 3.4. Let $q+1=\frac{n p}{n-p}$, the Sobolev critical exponent. Let $u$ be a nonnegative weak supersolution of (1.1). Let $\Omega^{\prime}$ be a subdomain contained compactly in $\Omega$. Let $0 \leq \rho \leq \frac{\operatorname{dist}\left(\Omega^{\prime}, \partial \Omega\right)}{4}$ and $t_{0} \in(0, T]$. Assume that

$$
\left|\Omega^{\prime} \cap\left\{u\left(t_{0}\right) \geq L\right\}\right| \geq \alpha\left|\Omega^{\prime}\right|
$$


holds for some $L>0$ and $\alpha \in(0,1]$. Then there exist positive integer $N=N\left(\Omega^{\prime}\right)$, positive numbers $\delta<1, \eta<1$ and positive integers $I$ and $J$ depending on $p, n, \alpha$ and independent of $L$, and a time $t_{N}>t_{0}$ such that

$$
u \geq \eta L
$$

almost everywhere in

$$
\Omega^{\prime} \times\left(t_{N}+\left(k+\frac{1}{2}\right) \frac{\delta(\eta L)^{q+1-p}}{2^{J+I}} \rho^{p}, t_{N}+(k+1) \frac{\delta(\eta L)^{q+1-p}}{2^{J+I}} \rho^{p}\right)
$$

for some $k \in\left\{0,1, \ldots, 2^{J+I}-1\right\}$, where $t_{N}$ is written as

$$
t_{N}=t_{0}+\left(\ell+\frac{3}{4}\right) \frac{\delta(\eta L)^{q+1-p}}{2^{J+I}} \rho^{p}
$$

for some $\ell \in\left\{0,1, \ldots, 2^{J+I-1}\right\}$.

If a nonnegative weak supersolution $u$ is positive in $\Omega$ at some time $t_{0} \geq 0$, its positivity may expand in a future time interval starting from $t_{0}$, that is without any waiting time. See the proof in [15, Corollary 4.8].

Corollary 3.5. Let $q+1=\frac{n p}{n-p}$, the Sobolev critical exponent. Let $u$ be a nonnegative weak supersolution of (1.1). Let $\Omega$ be a subdomain contained compactly in $\Omega$. Suppose that $u\left(t_{0}\right)>0$ almost everywhere in $\Omega^{\prime}$ for some $t_{0} \in[0, T)$. Then there exist positive numbers $\eta_{0}$ and $\tau_{0}$ such that

$$
u \geq \eta_{0} \text { a.e. in } \Omega^{\prime} \times\left(t_{0}, t_{0}+\tau_{0}\right) .
$$

Once the interior positivity holds true, the positivity around the boundary can follow from the usual comparison function. See the proof in [15, Proposition 4.9].

Proposition 3.6. (Positivity of the solution near the boundary). If $u_{0}>0$ in $\Omega$ then every nonnegative weak supersolution $u$ to Eqn (1.1) is positive near the boundary.

\subsection{Extinction of solutions}

In this section, we will state the definition of finite extinction time and a proposition which ensures the existence of finite extinction time of a solution to Eqn (1.1). Firstly, the extinction time is defined as follows:

Definition 3.7. (Extinction time). Let $u$ be a nonnegative weak solution to Eqn (1.1) in $\Omega_{\infty}$. We call a positive number $T^{*}$ an extinction time of $u$ if

(1) $u(x, t)$ is nonnegative and not identically zero on $\Omega \times\left(0, T^{*}\right)$.

(2) $u(x, t)=0$ for any $x \in \bar{\Omega}$ and for all $t \geq T^{*}$

The following proposition presents the finite time extinction of the solution of (1.1).

Proposition 3.8. Let $q+1=\frac{n p}{n-p}$, the Sobolev critical exponent. Suppose the initial data $u_{0}>0$ in $\Omega$. Then there exists $T^{*}>0$ such that $u=0$ in $\Omega \times\left[T^{*}, \infty\right)$

For the proof see Appendix. Here we use the special function peculiar to the Sobolev critical case $q+1=\frac{n p}{n-p}$. 
AJMS 28,1

Proposition 3.9. Let $q+1=\frac{n p}{n-p}$, the Sobolev critical exponent. Let $u$ be a nonnegative weak solution to (1.1) in $\Omega_{\infty}$. Then there exists an extinction time $T^{*}>0$ for $u$ which is bounded as

$$
T^{*} \leq \frac{q}{q+1-p}\left(\frac{\max _{\Omega} u_{0}}{\min _{\Omega} Y}\right)^{q+1-p},
$$

where $Y$ is Talenti's function defined by

$$
Y(x)=Y_{\lambda, y}(x):=\frac{1}{\lambda}\left(n\left(\frac{n-p}{p-1}\right)^{p-1}\right)^{\frac{1}{p}}\left(1+\left(\frac{|x-y|}{\lambda}\right)^{\frac{p}{p-1}}\right)^{-1}
$$

with a positive parameter $\lambda$.

Talenti's function is an unique solution of the stationary equation on all of space $\mathbb{R}^{n}$ corresponding to $(1.1)$ (see $[16,17])$.

\section{Main theorem}

Our main result in this paper is the following theorem.

Theorem 4.1. Suppose that $n=3$. Let $p \in[2,3)$ and $q+1=\frac{3 p}{3-p}$, the Sobolev critical exponent. Let $\Omega$ be a convex bounded domain with smooth boundary. Suppose that the initial data $u_{0}$ belong to $W_{0}^{1, p}(\Omega)$, positive and bounded in $\Omega$. Let $u$ be a continuous weak solution of Eqn (1.1) in $\Omega_{\infty}:=\Omega \times(0, \infty)$ with the initial and boundary data $u_{0}$. Then there exists a positive number $T<\infty$ such that $u>0$ in $\Omega \times[0, \mathrm{~T})$ and $u=0$ in $\Omega \times[\mathrm{T}, \infty)$.

Proof. By Theorem 3.4 and Proposition 3.8, we have the existence of finite positive $T_{0}$ and $T^{*}$ such that $u>0$ in $\Omega \times\left[0, T_{0}\right)$ and $u=0$ in $\Omega \times\left[T^{*}, \infty\right)$. We notice that the solution $u$ may have a positive portion and a zero one in $\Omega \times\left[T_{0}, T^{*}\right)$. Therefore, our aim is to show that $T_{0}=T^{*}$. The uniqueness of a weak solution to (1.1) holds true by the comparison principle, Theorem 3.3. Thus, we may assume the following: for any $t_{0} \in\left[T_{0}, T^{*}\right)$, there exists a space point $x_{0} \in \Omega$ such that $u\left(x_{0} ; t_{0}\right)>0$. Indeed, if $u\left(t_{0}\right)=0$ in $\Omega$ for some $t_{0} \in\left[T_{0}, T^{*}\right)$, then the function $u$ being extended to zero in $\Omega \times\left[t_{0}, \infty\right)$ is also a weak solution of Eqn (1.1). That contradicts the choice of $t_{0} \in\left[T_{0}, T^{*}\right)$ (see Figures 1 and 2).

For any $t_{0} \in\left[T_{0}, T^{*}\right)$, let $x_{0} \in \Omega$ such that $u\left(x_{0}, t_{0}\right)>0$. Since $u$ is continuous, there exists a ball $B_{\rho_{0}}\left(x_{0}\right) \subset \Omega$ with center $x_{0}$ and radius $\rho_{0}>0$ and a positive number $\delta_{0}$ such that $u>0$ in $B_{\rho_{0}}\left(x_{0}\right) \times\left(t_{0}, t_{0}+\delta_{0}\right)$. To proceed our argument, we will work under the polar coordinates around any boundary point of $\Omega$. Let $x_{1}$ be any point on $\partial \Omega$. By a translation, let $x_{1}$ be transformed to the origin. We use the polar coordinates around the origin, where Eqn (1.1) is invariant under a parallel transformation and a rotation and thus, if necessary, by the rotation around the origin, we may assume that the first component axis is the line passing through two points, the origin and $x_{0}$, and the other component axes are orthogonal to the above first axis and each other. Then, we make some conic space region with vertex at the origin and small angles around the first axis such that the final arc like part of the cone is in $B_{\rho_{0}}\left(x_{0}\right)$. This conic space region is denoted by $\mathcal{C}$ and, let $\mathcal{R}:=\mathcal{C} \times\left(t_{0}, t_{0}+\delta_{0}\right)$. It is verified by the convexity of the domain that $\mathcal{C} \subset \Omega$, if the angles around the first axis are small, and thus, $\mathcal{R} \subset \Omega \times\left[T_{0}, T^{*}\right)$ for a small positive $\delta_{0}$. 


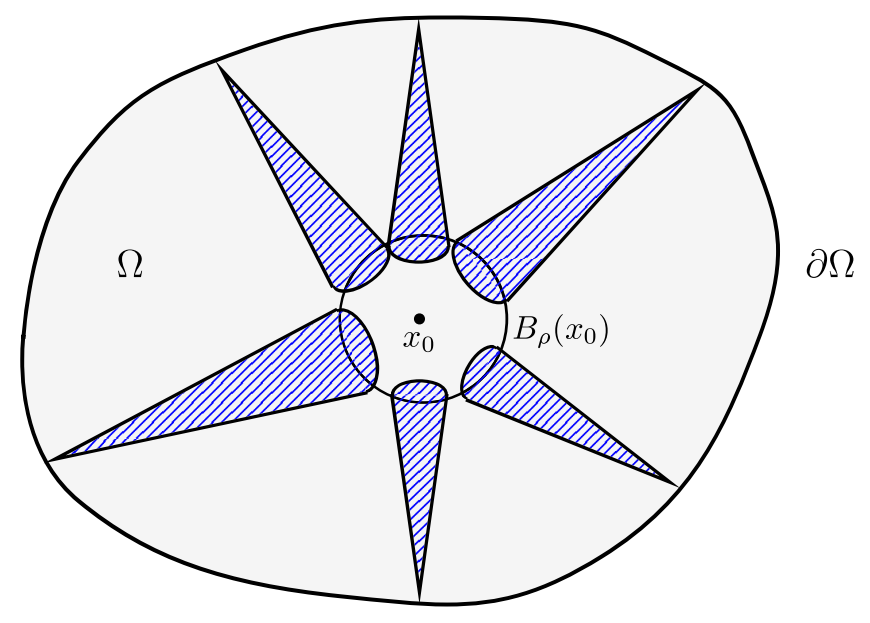

Equation of fast diffusion

Figure 1. Domain with boundary and conic region

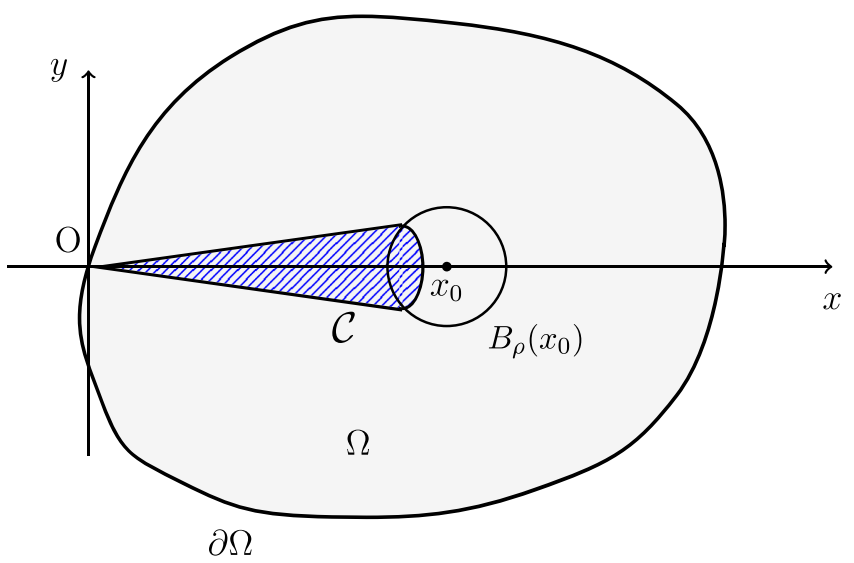

Figure 2. coordinate axis $x$ and $y$

Let the comparison function in the three dimension be defined as

$$
w(r, \theta, \phi, t):=m\left(t-t_{0}\right) r^{\mu} \cos \left(\frac{\pi \theta}{2 \alpha}\right) \sin \left(\frac{\pi}{2 \beta}\left(\phi-\frac{\pi}{2}\right)+\frac{\pi}{2}\right)
$$

in the time-space region $\mathcal{R}:=\mathcal{C} \times\left(t_{0}, t_{0}+\delta_{0}\right)$ given by the variables $(r, \theta, \phi, t)$ in the range

$$
\left\{\begin{array}{l}
0<r<R:=\operatorname{diam}(\Omega), \\
\frac{\pi}{2}-\beta<\phi<\frac{\pi}{2}+\beta, \\
-\alpha<\theta<\alpha \\
t_{0}<t<t_{0}+\delta_{0},
\end{array}\right.
$$


AJMS

28,1

52

Figure 3.

Graph of $y=\cos \left(\frac{\pi \theta}{2 \alpha}\right)$

Figure 4.

Graph of $y=r^{\mu}$

Figure 5.

Graph of

$y=\sin$

$\left(\frac{\pi}{2 \beta}\left(\phi-\frac{\pi}{2}\right)+\frac{\pi}{2}\right)$ where the positive parameters $\alpha, \beta, \delta_{0}, m$ and $\mu$ are determined according to the demand, later. As before, we choose $\alpha, \beta$ and $\delta_{0}$ so small that $\mathcal{R} \subset \Omega \times\left[T_{0}, T^{*}\right)$. Again, that is possible by the hypothesis that the domain $\Omega$ is convex (see Figures $3-5$ ).

For brevity, we use the abbreviation hereafter

$$
I=\frac{\pi \theta}{2 \alpha}, \quad I I=\frac{\pi}{2 \beta}\left(\phi-\frac{\pi}{2}\right)+\frac{\pi}{2} .
$$
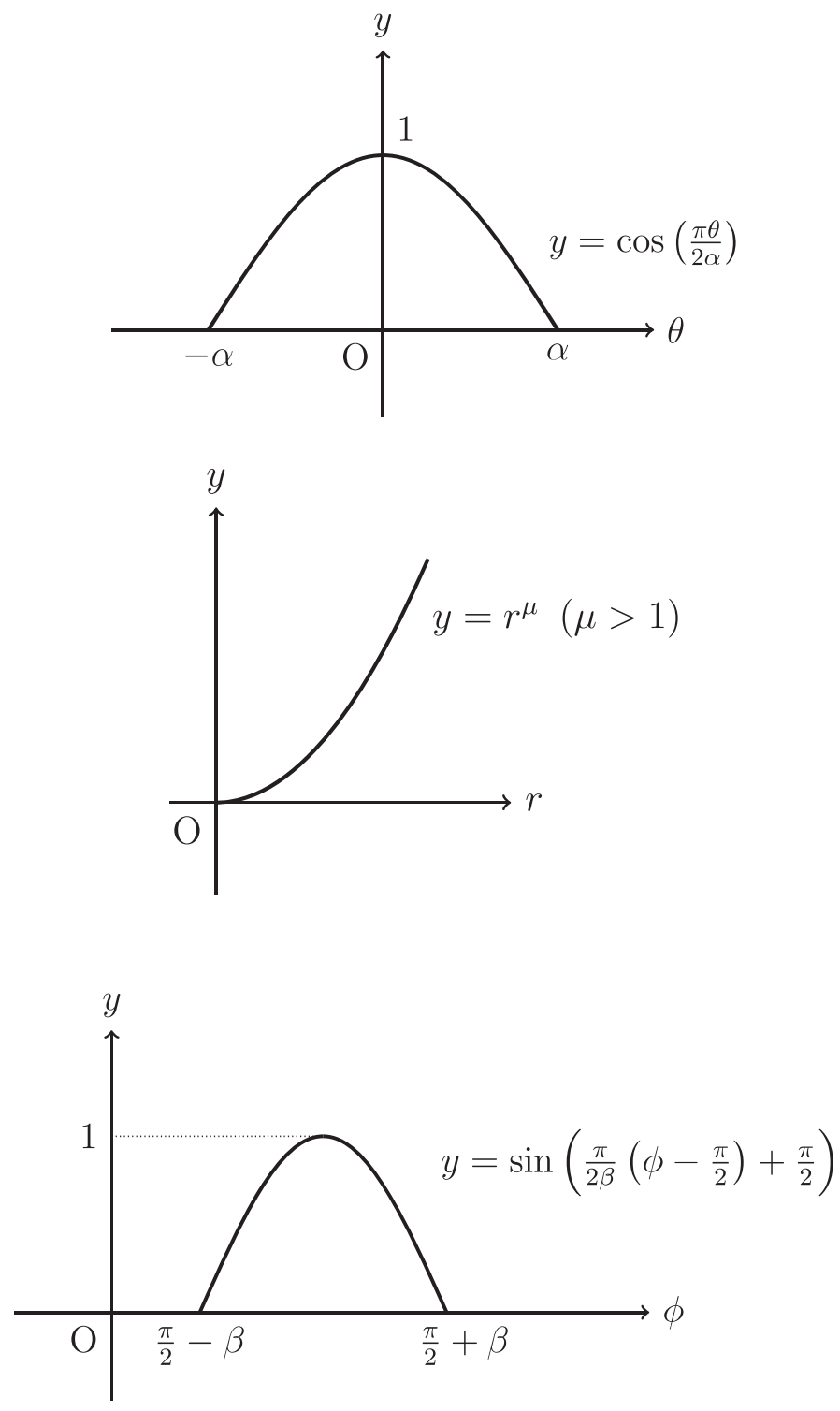
There holds

$$
\begin{aligned}
& |\nabla w|^{2}=m^{2}\left(t-t_{0}\right)^{2} r^{2 \mu-2}\left[\mu^{2} \cos ^{2}(I) \sin ^{2}(I I)+\left(\frac{\pi}{2 \beta}\right)^{2} \cos ^{2}(I) \cos ^{2}(I I)\right. \\
& \left.+\frac{1}{\sin ^{2} \phi}\left(\frac{\pi}{2 \alpha}\right)^{2} \sin ^{2}(I) \sin ^{2}(I I)\right] ; \\
& \Delta w=w_{r r}+\frac{1}{r^{2}} w_{\phi \phi}+\frac{1}{r^{2} \sin ^{2} \phi} w_{\theta \theta}+\frac{2}{r} w_{r}+\frac{\cot \phi}{r^{2}} w_{\phi} \\
& \quad=m\left(t-t_{0}\right) r^{\mu-2}\left[\left\{\mu^{2}-\left(\frac{\pi}{2 \beta}\right)^{2}+\mu-\frac{1}{\sin ^{2} \phi}\left(\frac{\pi}{2 \alpha}\right)^{2}\right\} \cos (I) \sin (I I)\right. \\
& \left.+\left(\frac{\pi}{2 \beta}\right) \frac{\cos \phi}{\sin \phi} \cos (I) \cos (I I)\right] .
\end{aligned}
$$

We know that

$$
\Delta_{p} w=|\nabla w|^{p-4}\left[|\nabla w|^{2} \Delta w+(p-2) S\right]
$$

where

$$
S=\sum_{i, j=1}^{n} w_{x_{i}} w_{x_{j}} w_{x_{i} x_{j}}
$$

The drift term is computed as

$$
\begin{aligned}
S= & m^{3}\left(t-t_{0}\right)^{3} r^{3 \mu-4}\left[\left(\mu^{4}-\mu^{3}\right) \cos ^{3}(I) \sin ^{3}(I I)\right. \\
& +2 \mu\left(\frac{\pi}{2 \beta}\right)^{2} \cos ^{3}(I) \sin (I I) \cos ^{2}(I I) \\
& -\left(\frac{\pi}{2 \beta}\right)^{4} \cos ^{3}(I) \sin (I I) \cos ^{2}(I I) \\
& +\frac{2}{\sin ^{2} \phi} \mu^{2}\left(\frac{\pi}{2 \alpha}\right)^{2} \sin ^{2}(I) \cos (I) \sin ^{3}(I I) \\
& +\frac{2}{\sin ^{2} \phi}\left(\frac{\pi}{2 \alpha}\right)^{2}\left(\frac{\pi}{2 \beta}\right)^{2} \sin ^{2}(I) \cos (I) \sin (I I) \cos ^{2}(I I) \\
& -\frac{1}{\sin ^{4} \phi}\left(\frac{\pi}{2 \alpha}\right)^{4} \sin ^{2}(I) \cos (I) \\
& -\mu \sin ^{3}(I I)\left(\frac{\pi}{2 \beta}\right)^{2} \cos ^{3}(I) \sin (I I) \cos ^{2}(I I) \\
& -\frac{1}{\sin ^{2} \phi} \mu\left(\frac{\pi}{2 \alpha}\right)^{2} \sin ^{2}(I) \cos (I) \sin ^{3}(I I) \\
& \left.-\frac{\cos ^{3} \phi}{\sin ^{3} \phi}\left(\frac{\pi}{2 \alpha}\right)^{2}\left(\frac{\pi}{2 \beta}\right) \sin ^{2}(I) \cos (I) \sin ^{2}(I I) \cos (I I)\right]
\end{aligned}
$$

Equation of fast diffusion 
AJMS

28,1

54 and thus,

$$
\begin{aligned}
& S=m^{3}\left(t-t_{0}\right)^{3} r^{3 \mu-4} \cos (I) \sin (I I)\left[\left(\mu^{4}-\mu^{3}\right) \cos ^{2}(I) \sin ^{2}(I I)\right. \\
& +2 \mu^{2}\left(\frac{\pi}{2 \beta}\right)^{2} \cos ^{2}(I) \cos ^{2}(I I)-\left(\frac{\pi}{2 \beta}\right)^{4} \cos ^{2}(I) \cos ^{2}(I I) \\
& +\frac{2}{\sin ^{2} \phi} \mu^{2}\left(\frac{\pi}{2 \alpha}\right)^{2} \sin ^{2}(I) \sin ^{2}(I I)+\frac{2}{\sin ^{2} \phi}\left(\frac{\pi}{2 \alpha}\right)^{2}\left(\frac{\pi}{2 \beta}\right)^{2} \sin ^{2}(I) \cos ^{2}(I I) \\
& -\frac{1}{\sin ^{4} \phi}\left(\frac{\pi}{2 \alpha}\right)^{4} \sin ^{2}(I) \sin ^{2}(I I)-\mu\left(\frac{\pi}{2 \beta}\right)^{2} \cos ^{2}(I) \cos ^{2}(I I) \\
& -\frac{1}{\sin ^{2} \phi} \mu\left(\frac{\pi}{2 \alpha}\right)^{2} \sin ^{2}(I) \sin ^{2}(I I) \\
& \left.-\frac{\cos \phi}{\sin ^{3} \phi}\left(\frac{\pi}{2 \alpha}\right)^{2}\left(\frac{\pi}{2 \beta}\right) \sin ^{2}(I) \sin (I I) \cos (I I)\right] \\
& \geq m^{3}\left(t-t_{0}\right)^{3} r^{3 \mu-4} \cos (I) \sin (I I)\left[\left(\mu^{4}-\mu^{3}\right) \cos ^{2}(I) \sin ^{2}(I I)\right. \\
& +\left(\frac{\pi}{2 \beta}\right)^{2}\left(2 \mu^{2}-\left(\frac{\pi}{2 \beta}\right)^{2}-\mu\right) \cos ^{2}(I) \cos ^{2}(I I) \\
& +\frac{1}{\sin ^{2} \phi}\left(\mu^{2}-\frac{\left(\frac{\pi}{2 \alpha}\right)^{2}}{\sin ^{2} \phi}-\mu\right)\left(\frac{\pi}{2 \alpha}\right)^{2} \sin ^{2}(I) \sin ^{2}(I I) \\
& +\frac{2}{\sin ^{2} \phi}\left(\frac{\pi}{2 \alpha}\right)^{2}\left(\frac{\pi}{2 \beta}\right)^{2} \sin ^{2}(I) \\
& \left.-\frac{\cos \phi}{\sin ^{3} \phi}\left(\frac{\pi}{2 \alpha}\right)^{2}\left(\frac{\pi}{2 \beta}\right) \sin ^{2}(I) \sin (I I) \cos (I I)\right] \\
& =m^{3}\left(t-t_{0}\right)^{3} r^{3 \mu-4} \cos (I) \sin (I I)\left[\left(\mu^{4}-\mu^{3}\right) \cos ^{2}(I) \sin ^{2}(I I)\right. \\
& +\left(\frac{\pi}{2 \beta}\right)^{2}\left(2 \mu^{2}-\left(\frac{\pi}{2 \beta}\right)^{2}-\mu\right) \cos ^{2}(I) \cos ^{2}(I I) \\
& +\frac{1}{\sin ^{2} \phi}\left(\mu^{2}-\frac{\left(\frac{\pi}{2 \alpha}\right)^{2}}{\sin ^{2} \phi}-\mu\right)\left(\frac{\pi}{2 \alpha}\right)^{2} \sin ^{2}(I) \sin ^{2}(I I) \\
& \left.+\frac{1}{\sin ^{2} \phi}\left(\frac{\pi}{2 \alpha}\right)^{2}\left(\frac{\pi}{2 \beta}\right) \sin ^{2}(I)\left\{2\left(\frac{\pi}{2 \beta}\right)-\frac{\cos \phi}{\sin \phi} \sin (I I) \cos (I I)\right\}\right] \geq 0
\end{aligned}
$$


if we choose as $\frac{\mu^{2}}{2} \geq\left(\frac{\pi}{2 \beta}\right)^{2}$ which is verified by a large positive $\mu$ depending on a small $\beta$, and the lower positive bound of $\sin \phi$ such that $\frac{1}{2} \leq \sin \phi \leq 1$ for $\phi \in\left(\frac{\pi}{2}-\beta, \frac{\pi}{2}+\beta\right)$.

$$
\begin{gathered}
|\nabla w|^{p-4}=m^{p-4}\left(t-t_{0}\right)^{p-4} r^{(p-4)(\mu-1)}\left[\mu^{2} \cos ^{2}(I) \sin ^{2}(I I)\right. \\
\left.+\left(\frac{\pi}{2 \beta}\right)^{2} \cos ^{2}(I) \cos ^{2}(I I)+\frac{1}{\sin ^{2} \phi}\left(\frac{\pi}{2 \alpha}\right)^{2} \sin ^{2}(I) \sin ^{2}(I I)\right]^{\frac{p-4}{2}} \geq 0
\end{gathered}
$$

and thus, letting $L w=\partial_{t} w^{q}-\Delta_{p} w$, we have

$$
\begin{aligned}
& -L w \geq-\partial_{t} w^{q}+|\nabla w|^{p-2} \Delta w \\
& =-q m^{q}\left(t-t_{0}\right)^{q-1} r^{\mu q} \cos ^{q}(I) \sin ^{q}(I I)+m^{p-1}\left(t-t_{0}\right)^{p-1} r^{(\mu-1)(p-2)} \\
& \times\left[\mu^{2} \cos ^{2}(I) \sin ^{2}(I I)+\left(\frac{\pi}{2 \beta}\right)^{2} \cos ^{2}(I) \cos ^{2}(I I)\right. \\
& \left.+\frac{1}{\sin ^{2} \phi}\left(\frac{\pi}{2 \alpha}\right)^{2} \sin ^{2}(I) \sin ^{2}(I I)\right]^{\frac{p-2}{2}} \\
& \times r^{\mu-2}\left[\left(\mu^{2}-\left(\frac{\pi}{2 \beta}\right)^{2}+\mu-\frac{1}{\sin ^{2} \phi}\left(\frac{\pi}{2 \alpha}\right)^{2}\right) \cos (I) \sin (I I)\right. \\
& \left.+\left(\frac{\pi}{2 \beta}\right) \frac{\cos \phi}{\sin \phi} \cos (I) \cos (I I)\right] \\
& \geq-q m^{q}\left(t-t_{0}\right)^{q-1} r^{\mu q} \cos ^{q}(I) \sin ^{q}(I I) \\
& +m^{p-1}\left(t-t_{0}\right)^{p-1} r^{\mu(p-1)-p} \mu^{p-2} \cos ^{p-2}(I) \sin ^{p-2}(I I) \\
& \times\left[\left(\mu^{2}-\left(\frac{\pi}{2 \beta}\right)^{2}+\mu-\frac{1}{\sin ^{2} \phi}\left(\frac{\pi}{2 \alpha}\right)^{2}\right) \cos (I) \sin (I I)\right] \\
& \geq m^{p-1}\left(t-t_{0}\right)^{p-1} r^{\mu(p-1)-p} \cos ^{p-1}(I) \sin ^{p-1}(I I) \\
& \times\left[-q m^{q-(p-1)}\left(t-t_{0}\right)^{q-p} r^{\mu(q-p+1)+p} \cos ^{(q-p+1)}(I) \sin ^{(q-p+1)}(I I)\right. \\
& \left.+\mu^{p-2}\left(\mu^{2}-\left(\frac{\pi}{2 \beta}\right)^{2}+\mu-\frac{1}{\sin ^{2} \phi}\left(\frac{\pi}{2 \alpha}\right)^{2}\right)\right] \\
& \geq m^{p-1}\left(t-t_{0}\right)^{p-1} r^{\mu(p-1)-p} \cos ^{p-1}(I) \sin ^{p-1}(I I) \\
& \times\left[-q m^{q-(p-1)}\left(t-t_{0}\right)^{q-p} R^{\mu(q-p+1)+p}\right. \\
& \left.+\mu^{p-2}\left(\mu^{2}-\left(\frac{\pi}{2 \beta}\right)^{2}+\mu-\frac{1}{\sin ^{2} \phi}\left(\frac{\pi}{2 \alpha}\right)^{2}\right)\right] \geq 0 .
\end{aligned}
$$

Here the reasoning is as follows: since $0 \leq r \leq R=\operatorname{diam}(\Omega)$ and $t_{0} \leq t \leq t_{0}+\delta_{0}$, the parameter $m$ can be so small that the quantity in the bracket is positive. Thus, $L w=\partial_{t} w^{q}-\Delta_{p} w \leq 0=L u$ in $\mathcal{R}$. The boundary condition of $w$ is verified as follows: On the lateral boundary, $w=0$ because at $\theta=\alpha,-\alpha$,
Equation of fast diffusion 
and at $\phi=\frac{\pi}{2}-\beta, \frac{\pi}{2}+\beta$,

$$
\frac{\pi}{2 \beta}\left(\phi-\frac{\pi}{2}\right)+\frac{\pi}{2}=0, \pi ; \quad \sin (I I)=0 .
$$

On the arc like boundary of $\mathcal{R}, w \leq u$ if the parameter $m$ is so small that $0 \leq w \leq m \delta_{0}$ $(\operatorname{diam}(\Omega))^{\mu} \leq \min _{\mathcal{A}} u$, where $\mathcal{A}=B_{\rho_{0}}\left(x_{0}\right) \times\left(t_{0}, t_{0}+\delta_{0}\right)$. On the initial boundary $\mathcal{C} \times$ $\left\{t=t_{0}\right\}, w\left(x, t_{0}\right)=0 \leq u\left(x, t_{0}\right)$. Therefore, $w$ is the subsolution of $L$ in $\mathcal{R}$ and thus, $u \geq w$ in $\mathcal{R}$ by Theorem 3.3. Hence, the solution $u$ is positive in $\mathcal{R}$. This is true for any $\mathcal{R}$ with vertex on the boundary $\partial \Omega$ and thus, $u$ is positive in $\Omega \times\left(t_{0}, t_{0}+\delta_{0}\right)$, because of the convexity of the domain. Hence the proof is complete.

\section{References}

[1] DiBenedetto E. Continuity of weak solutions to a general porous medium equation. Indiana Univ Math J. 1983; 32: 83-118.

[2] Sabanina ES. A class of non-linear degenerate parabolic equations. Soviet Math Dokl. 1962; 143: 495-98.

[3] Sacks P. Continuity of solution of a singular parabolic equation, Nonlinear Anal. 1983; 7: 387-409.

[4] Benilan P, Crandall MG. The continuous dependence on $\phi$ of solutions of $u_{t}-\Delta \phi(u)=0$, Indiana Univ Math J. 1981; 30: 161-76.

[5] Diaz G, Diaz I. Finite extinction time for a class of non linear parabolic equations, Comm Partial Diff Eq. 1979; 4: 1213-31.

[6] Kwong YC. Interior and boundary regularity of solutions to a plasma type equation, Proc Am Math Soc. 1988; 104(2): 472-78.

[7] Berryman JG, Holland CJ. Stability of the separable solution for fast diffusion, Arch Rational Mech Anal. 1980.

[8] Kwong YC. Asymptotic behavior of a plasma type equation with finite extinction, Arch. Rational Mech Anal. 1988; 104(3): 277-94.

[9] Kwong YC. The asymptotic behavior of the plasma equation with homogeneous Dirichlet boundary condition and non-negative initial data, Appl Anal. 1988; 28(2): 95-113.

[10] DiBenedetto E. Degenerate parabolic equations, New York, NY: Universitext, SpringerVerlag; 1993.

[11] DiBenedetto E, Gianazza U, Vespri V. Harnack's inequality for degenerate and singular parabolic equations, Springer Monograph Math. 2012.

[12] Kuusi T, Siljander J, Urbano JM. Local Hölder continuity for doubly nonlinear parabolic equations, Indiana Univ Math J. 2012; 61(1): 399-430.

[13] Kuusi T, Misawa M, Nakamura K, Global existence for the p-Sobolev flow, J Diff Equat. 2021; 279: $245-81$.

[14] Nakamura K, Misawa M. Existence of weak solution to the p-Sobolev flow, Nonlinear Anal. 2018; 175: $157-72$.

[15] Kuusi T, Misawa M, Nakamura K. Regularity estimates for the p-Sobolev flow, J Geom Anal. 2020; 30(2): 1918-64.

[16] Sciunzi B. Classification of positive $\mathcal{D}^{1, p}\left(\mathbb{R}^{N}\right)$ - solution to the critical $p$-Laplace equation, Adv Math. 2016; 291: 12-23.

[17] Talenti G. Best constant in Sobolev inequality, Ann Mat Pura Appl. 1976; 110(4): 353-72. 
[18] Misawa M, Nakamura K, Sarkar H. A finite time extinction profile and optimal decay for a fast diffusive doubly nonlinear equation, preprint.

[19] Evans LC, Partial differential equations, Providence, RI: American Mathematical Society; 1998.

[20] Ladyzenskaja OA, Solonnikov VA, Ural'ceva NN. Linear and quasilinear equations of parabolic type, Math Mono. 1968; 23, Am Math Soc., Providence, R, I.

\section{Appendix}

Proofs of Theorem 3.3 and of Proposition 3.9

Here we are going to provide a detailed proof of Theorem 3.3, and Propositions 3.8 and 3.9, since their results are actually used in the proof of the main theorem.

At first we will depict the proof of Theorem 3.3.

Proof of Theorem 3.3. Following [15], we prove our assertion. For a small $\delta>0$, let us define the Lipschitz function $\phi_{\delta}$ by

$$
\phi_{\delta}(x):=\min \left\{1, \frac{x_{+}}{\delta}\right\}
$$

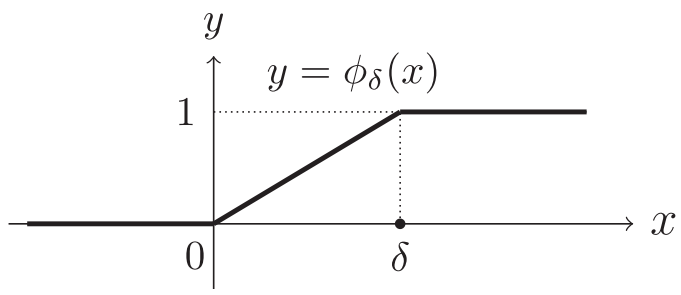

Note that $\phi_{\delta}(v-u) \in L^{\infty}\left(\Omega_{T}\right)$ and $L^{\infty}\left(0, T ; W_{0}^{1, p}(\Omega)\right)$. Let $0<t_{1}<t \leq T$ and $\sigma_{t_{1}, t}$ be the Lipschitz cut off function on time such that

$$
0 \leq \sigma_{t_{1}, t} \leq 1, \sigma_{t_{1}, t}=1 \operatorname{in}\left(t_{1}+\delta, t-\delta\right) \text { and } \operatorname{supp}\left(\sigma_{t_{1}, t}\right) \subset\left(t_{1}, t\right) .
$$

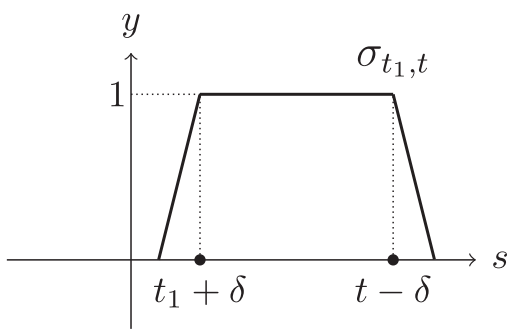

Choose an admissible test function $\sigma_{t_{1}, t} \phi_{\delta}(v-u)$ to have

$$
\int_{\Omega_{t_{1}, t}} \partial_{t}\left(u^{q}\right) \sigma_{t_{1}, t} \phi_{\delta}(v-u) d x d t+\int_{\Omega_{t_{1}, t}}|\nabla u|^{p-2} \nabla u \cdot \nabla\left(\phi_{\delta}(v-u)\right) \sigma_{t_{1}, t} d x d t \geq 0
$$

Equation of fast diffusion 
AJMS

28,1

58

and

$$
\int_{\Omega_{t_{1}, t}} \partial_{t}\left(v^{q}\right) \sigma_{t_{1}, t} \phi_{\delta}(v-u) d x d t+\int_{\Omega_{t_{1}, t}}|\nabla v|^{p-2} \nabla v \cdot \nabla\left(\phi_{\delta}(v-u)\right) \sigma_{t_{1}, t} d x d t \leq 0 .
$$

Note that

$$
\nabla\left(\phi_{\delta}(v-u)\right)= \begin{cases}\frac{1}{\delta}(\nabla v-\nabla u) & 0<v-u<\delta \\ 0 & \text { otherwise }\end{cases}
$$

and thus,

$$
\nabla\left(\phi_{\delta}(v-u)\right)=\frac{1}{\delta}(\nabla v-\nabla u) \chi_{\{0<v-u<\delta\}} .
$$

Subtracting (A.1) from (A.2) to have

$$
\begin{aligned}
& \int_{\Omega_{t_{1}, t}} \partial_{t}\left(v^{q}-u^{q}\right) \sigma_{t_{1}, t} \phi_{\delta}(v-u) d x d t+\int_{\Omega_{t_{1}, t}}\left(|\nabla v|^{p-2} \nabla v\right. \\
& \left.-|\nabla u|^{p-2} \nabla u\right) \frac{1}{\delta} \times(\nabla v-\nabla u) \chi_{\{0<v-u<\delta\}} \sigma_{t_{1}, t} d x d t \leq 0
\end{aligned}
$$

by (2.1) in Lemma 2.1, the second term on the left hand side of (A.3) is bounded below as

$$
\frac{C}{\delta} \int_{\Omega_{t_{1}, t}}(|\nabla v-\nabla u|)^{p} \chi_{\{0<v-u<\delta\}} \sigma_{t_{1}, t} d x d t \geq 0
$$

for a positive constant $C$. Thus (A.3) and (A.4) lead to

$$
\int_{\Omega_{t_{1}, t}} \partial_{t}\left(v^{q}-u^{q}\right) \phi_{\delta}(v-u) \sigma_{t_{1}, t} d x d t \leq 0 .
$$

Since $\partial_{t}\left(v^{q}\right)$ and $\partial_{t}\left(u^{q}\right)$ belong to $L^{2}\left(\Omega_{T}\right)$, by the Lebesgue dominated convergence theorem, we can take the limit as $\delta \searrow 0$ in (A.5) and then obtain, as $t_{1} \searrow 0$,

$$
\int_{\Omega}\left(v^{q}(t)-u^{q}(t)\right)_{+} d x \leq 0
$$

and thus, $v^{q} \leq u^{q}$ in $\Omega$, for nonnegative $t \leq T$, which is equivalent to that $v(t) \leq u(t)$ in $\Omega$, for nonnegative $t \leq T$. Hence the proof is complete.

Proposition 3.8 is given by Proposition 3.9. Therefore, we are now going to exhibit the proof of the Proposition 3.9.

Proof of Proposition 3.9. The proof is similar to [13]. We consider the solution of the corresponding elliptic equation of (1.1) for the sake of construction of a suitable comparison function and this function is called Talenti function [17] which is defined as

$$
Y_{a, b y}(x):=\left(a+b|x-y|^{\frac{p}{p-1}}\right)^{-\frac{n-p}{p}}, \quad x, y \in \mathbb{R}^{n},
$$

where $a$ and $b$ are positive numbers. $G$. Talenti showed in [17] that this function is the best constant in Sobolev inequality. Furthermore, a straightforward mathematical calculation reveals that $Y_{a, b}$ is a solution of the equation

$$
-\Delta_{p} Y_{a, b, z}=n\left(\frac{n-p}{p-1}\right)^{p-1} a b^{p-1} Y_{a, b, z}^{q} \quad \text { in } \mathbb{R}^{n}
$$


In [16], Sciunzi showed that solution of this equation is necessarily of the form

$$
Y(x)=Y_{\lambda, y}(x):=\frac{1}{\lambda}\left(n\left(\frac{n-p}{p-1}\right)^{p-1}\right)^{\frac{1}{p}}\left(1+\left(\frac{|x-y|}{\lambda}\right)^{\frac{p}{p-1}}\right)^{-\frac{n-p}{p}}
$$

with a parameter $\lambda>0$. Now what remains to show that a solution of Eqn (1.1) should be extinct at a finite time.

To proceed further, we assume by a translation that the origin $0 \in \Omega$. Let $u=u(x, t)$ be a nonnegative weak solution to Eqn (1.1). Next, let $W(x, t)=X(x) T(t)$ be a nonnegative separable solution of

$$
\partial_{t} W^{q}-\Delta_{p} W=0 \text { in } \mathbb{R}^{n} \times(0, \infty) .
$$

Then $X(x) T(t)$ satisfies

$$
\begin{cases}\left(T(t)^{q}\right)^{\prime}=\mu T(t)^{p-1} & \text { in }(0, \infty) \\ \Delta_{p} X=\mu X^{q} & \text { in } \mathbb{R}^{n}\end{cases}
$$

where $\mu$ is a separation constant. Applying integration by parts to the first separable function, we see that the separation constant $\mu<0$. Let us set $X:=(-\mu)^{-\frac{1}{q+1-p}} Y$ to obtain

$$
-\Delta_{p} Y=Y^{q} \text { in } \mathbb{R}^{n}
$$

A finite-energy solution to (A.9) is given by (A.7). By direct computation, we find that

$$
T(t)=T(0)\left(1+\mu \frac{q+1-p}{q} T(0)^{p-(q+1)} t\right)_{+}^{\frac{1}{q+1-p}}
$$

solves the first equation in (A.8), where $T(0)$ is the initial data. Thus the vanishing time $T^{*}$ of $T(t)$ is given by

$$
T^{*}=(-\mu)^{-1} \frac{q}{q+1-p} T(0)^{q+1-p}
$$

Let $U(x, t)$ be $(-\mu)^{-\frac{1}{q+1-p}} Y(x) T(t)$. Then

$$
0=u(x, t) \leq U(x, t) \quad \text { on } \partial \Omega \times[0, T] .
$$

We choose the initial data for the ODE in (A.8) as

$$
T(0):=\frac{\max _{\Omega} u_{0}}{\min _{\Omega} Y}(-\mu)^{\frac{1}{q+1-p}}
$$

and therefore, we find that

$$
u_{0}(x) \leq U(x, 0) \quad \text { in } \Omega .
$$

According to Theorem 3.3, we have

$$
u(x, t) \leq U(x, t) \quad \text { in } \Omega_{T}
$$


AJMS

28,1

\section{0}

and thus, the vanishing time $T^{*}$ of $u(x, t)$ is estimated as

$$
T^{*} \leq T_{0}=\frac{q}{q+1-p}\left(\frac{\max _{\Omega} u_{0}}{\min _{\Omega} Y}\right)^{q+1-p},
$$

where (A.10) is used. The proof is complete.

\section{Corresponding author}

Md Abu Hanif Sarkar can be contacted at: sarkarhanif@ymail.com 